\title{
Residual Bond Graph Sinks for Numerical Evaluation of Analytical Redundancy Relations in Model Based Single Fault Detection and Isolation
}

\author{
W. Borutzky \\ Bonn-Rhein-Sieg University of Applied Sciences, D-53754 Sankt Augustin, Germany \\ borutzky@uni-koeln.de
}

\begin{abstract}
The paper proposes a bond graph approach to model based fault detection and isolation (FDI) that uses residual sinks. These elements couple a reference model of a process engineering system to a bond graph model of the system that is subject to disturbances caused by faults. In this paper it is assumed that two faults do not appear simultaneously. The underlying mathematical model is a Differential Algebraic Equations (DAE) system. The approach is illustrated by means of the often used hydraulic two tanks system.
\end{abstract}

Keywords-Bond Graph models for fault detection and isolation, analytical redundancy relations, residual sinks, DAE systems.

\section{INTRODUCTION}

With regard to safety, fault detection and isolation (FDI) is crucial in the supervision and closed loop control of all kinds of industrial process engineering systems. To that end, it is clear to compare the measured behaviour of a system with a reference and to use significant deviations as indicators to possible faults in some system components. The reference can be provided by a system model. The bond graph methodology [1, 2, 3, 4] is particularly suited for modelling multidisciplinary process engineering systems [5, 6]. Moreover, bond graph modelling has been intensively and successfully used for model based fault detection and isolation [7, 8, 9, 10, 11]. In these bond graph based approaches, derivation and evaluation of so-called Analytical Redundancy Relations (ARRs) play a central role. They establish constraints between known variables and include known model parameters. When a process operates under normal mode conditions, evaluation of the constraints should reveal values within certain small error bounds. Theoretically, these values, also called residuals, should be zero. Values exceeding given thresholds are indicators to faults in some system components. Structural analysis of the constraint relations reveals whether faults can be isolated or not.

On the other hand, artificial, so-called residual sinks have been added to bond graphs in order to support the tearing of the algebraic part of a Differential Algebraic Equations (DAE) system, to solve that part and to transform the DAE system into a state space model if possible (Borutzky and Cellier [12], Borutzky [13]). In this paper, it is shown how residual bond graph sinks can also be used for the numerical evaluation of ARRs. In contrast to their use in the context of tearing, the underlying mathematical model for fault detection and isolation remains a DAE system.

The paper is organised in the following manner. The next section recalls some relevant features of model based fault detection and isolation. Section III briefly reconsiders residual sinks and section IV presents the proposed bond graph approach to the numerical evaluation of residuals of ARRs in FDI. In section V, for illustration, the approach is applied to the often used example of a hydraulic two tanks system.
The underlying DAE system has been solved numerically by means of the open source mathematics software package Scilab [14]. The paper concludes by briefly summarising the features of the proposed bond graph approach.

\section{FAult Detection AND Isolation}

Fault detection and isolation starts from constraints between known variables. These relations also include known model parameters. Their numerical evaluation at each time instant reveals a residual. Under normal mode of operation it should be zero. In bond graphs, constraints are clearly indicated by junctions. Each junction contributes a continuity equation for flows or efforts respectively. By using the constitutive equations of bond graph elements and by elimination of unknown variables, ARRs may be obtained in symbolic form. The form of the set of ARRs is not unique and depends on the procedure. Moreover, algebraic dependencies indicated by causal paths in the bond graph and nonlinear constitutive relations may prevent the elimination of unknown variables. Given that unknown variables can be eliminated, then structural analysis of each equation reveals a so-called signature in terms of known variables and system component parameters. For illustration, consider the hydraulic two tanks system depicted in Fig. 1. Fig. 2 shows a causal bond graph of the system. The left hand side 0junction provides the continuity equation for the volume flow rates:

$$
0=Q_{p}-Q_{C_{1}}-Q_{R_{1}}
$$

If the left hand side zero is replaced by a residual, $\mathrm{res}_{1}$, and if the constitutive equations of the energy $\mathrm{C}$-store and of the hydraulic resistor are inserted, then the equation reads:

$$
\operatorname{res}_{1}=\mathrm{Q}_{\mathrm{p}}-\mathrm{C}_{1} \dot{\mathrm{p}}_{1}-\mathrm{k}_{1} \operatorname{sign}\left(\mathrm{p}_{1}-\mathrm{p}_{2}\right) \sqrt{\left|\mathrm{p}_{1}-\mathrm{p}_{2}\right|}
$$

Since the right hand side includes only known variables and known parameters, the equation clearly is an ARR. In this relation, tank $1, T_{1}$, contributes the parameter $C_{1}$ and the valve, $V_{1}$, between both tanks the parameter $k_{1}$. From this view of ARRs, an occurrence matrix can be set up with one row for each known variable or component parameter and one column for each residual. If the occurrence of a variable or a parameter in an ARR is indicated by ' 1 ' and its absence by ' 0 ', then the result is a binary matrix. This matrix is usually augmented by two other columns. The first one with the heading $\mathrm{D}$ indicates whether a fault can be detected. The second additional column with the heading I indicates whether a fault can be isolated [8]. If this is feasible, then it is marked by ' 1 ' otherwise by '0'. A fault can be detected if there is at least one non-zero entry in that row. 


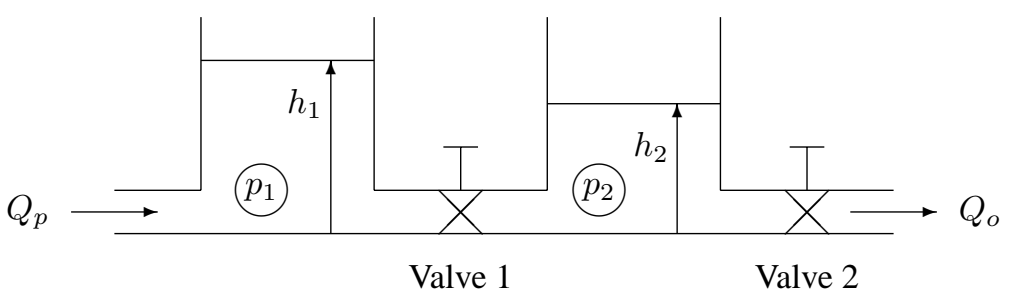

Fig. 1: Hydraulic two tanks system

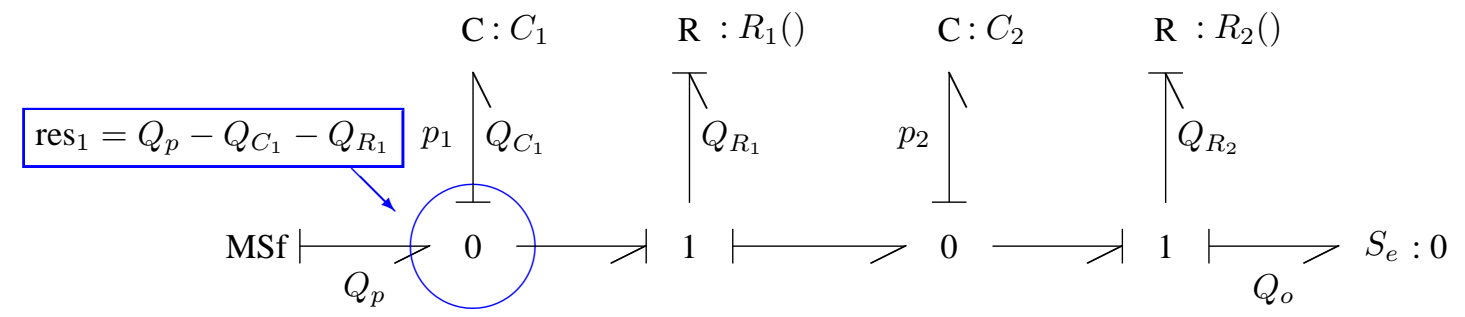

Fig. 2: Bond graph of the hydraulic two tanks system

\begin{tabular}{|c|l|c|c|c|c|}
\hline Component & Variable/Parameter & res $_{1}$ & res $_{2}$ & $\mathrm{D}$ & $\mathrm{I}$ \\
\hline Pump & $Q_{p}$ & 1 & 0 & 1 & 0 \\
\hline & $p_{1}$ & 1 & 1 & 1 & 0 \\
\hline & $p_{2}$ & 1 & 1 & 1 & 0 \\
\hline Tank 1 & $C_{1}=A_{T_{1}} /(\rho g)$ & 1 & 0 & 1 & 0 \\
\hline Tank 2 & $C_{2}=A_{T_{2}} /(\rho g)$ & 0 & 1 & 1 & 0 \\
\hline Valve 1 & $k_{1}=c_{d} A_{V_{1}} \sqrt{2 / \rho}$ & 1 & 1 & 1 & 0 \\
\hline Valve 2 & $k_{2}=c_{d} A_{V_{2}} \sqrt{2 / \rho}$ & 0 & 1 & 1 & 0 \\
\hline
\end{tabular}

TABLE I: Fault signature matrix of the two tanks systems

This is indicated by ' 1 ' in the first additional column, otherwise by ' 0 '. The augmented matrix is called fault signature matrix. Its columns are called fault signatures (of the ARRs of the residuals). Table I shows the fault signature matrix of the hydraulic two tanks system. The fault signature matrix of the two tanks system shows that none of the faults can be isolated. In fact, if, for a given time instant $t$, for instance, only residual $\mathrm{res}_{2}$ is above a given threshold, the reason may be either a leakage from the second tank or a fault in its outlet valve $V_{2}$. Clearly, a single fault can be located (isolated), if it can be detected and if the pattern of non-zero entries in the corresponding row of the matrix is unique.

Furthermore, the fault signature matrix includes only two residuals although also each 1 -junctions in the bond graph contributes a continuity equation for the efforts. However, their signature turns out to be identical to those already listed in the fault signature matrix. Thus, there are only two structurally independent residuals in the bond graph model of the two tanks system. In fact, consider, for instance, the sum of efforts at the 1-junction representing the volume flow rate, $Q_{R_{1}}$, through valve 1 between the two tanks. If the pressure drop across valve 1 is expressed by the known volume flow rate of the pump, $Q_{p}$, and the pressure in the first tank, $T_{1}$, then the residual, $\operatorname{res}_{3}$, of this 1 -junction reads:

$0=p_{1}-p_{2}-\frac{1}{k_{1}^{2}}\left(Q_{p}-C_{1} \dot{p}_{1}\right)^{2} \operatorname{sign}\left(\mathrm{Q}_{\mathrm{p}}-\mathrm{C}_{1} \dot{\mathrm{p}}_{1}\right)=\operatorname{res}_{3}$

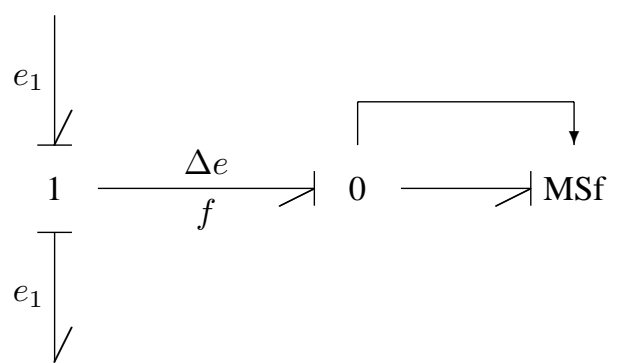

Fig. 3: Representation of a residual flow sink according to Bos 1986

Clearly, the signature of $\mathrm{res}_{3}$ is structurally identical to the one of res 1 . Consequently, it is of interest to derive a minimal number of structurally independent residuals from a bond graph model of a process engineering system.

\section{RESIDUAL BOND GRAPH SINKS}

A residual bond graph sink is a sink that adjusts its output power variable so that the power conjugated variable into the sink vanishes. Fig. 3 shows a graphical representation introduced by Bos [15]. It explicitly depicts the internal modulation of the sink. In this case, the difference of efforts, $\Delta e$, into the sink is sensed. It controls the output of the sink, $f$, such that the difference, $\Delta e$, vanishes. The difference $\Delta e$ can be considered the residual of the 1junction. For brevity we will denote residual sinks by $r S f$ or $r S e$, respectively. They can be used for different purposes, e. g., for representing internal constraint forces or moments in bodies [15, 4], or for indicating tearing variables in bond graph models (Borutzky [13]). In Karnopp's all derivative causalities approach to the derivation of Lagrange equations, residual flow sources indicate generalised coordinates. In that context they are know as artificial flow sources [16]. Furthermore, in [17], Gawthrop and Smith used residual sources for a modification of the standard sequential causality assignment procedure (SCAP) in the case of bond graphs with algebraic loops.

A residual sink results if the parameter of an energy store is assumed to tend to zero. A linear inertia in integral causal- 
ity with inertance I, for instance, provides a flow such that

$$
I \cdot \dot{f}=\Delta e
$$

For $I \rightarrow 0$, the constitutive equation of the I energy store turns into the trivial equation $0=\Delta e$ Now, the flow is no longer the output variable of an I energy store. It is not a state variable anymore. It is rather a variable determined by the algebraic equation $0=\Delta e$. As the derivative of $f$ has vanished, while $f$ itself is still an unknown, the mathematical model is a DAE system of index $\geq 1$ and the flow $f$ can be considered a component of a descriptor vector. The use of residual sinks can also be related to the method of singular perturbation. In the state equations of perturbed systems, the derivative of a fast component is multiplied by a parameter $\epsilon$ of very small value. Letting $\epsilon \rightarrow 0$, the ODEs turn into a DAE system.

\section{USING RESIDUAL BOND GRAPH SINKS FOR NumERICAL EVALUATIONS OF ARRS}

Fault detection and isolation is based on the comparison of the actual behaviour of a process engineering system with that of a reference model. Signals from the real engineering process can be obtained by means of sensors and can be fed into the reference model. However, the deliberate introduction of faults into the real process for test purposes may lead to hazardous situations, to periods of process instability if the equipment allows for introduction of faults at all. Therefore, it is obvious to replace the real process by a behavioural model which enables to introduce all kinds of faults without risk. In an offline simulation, the equations of the process model and of the reference model are solved simultaneously and residuals of ARRs, being indicators for faults, are numerically evaluated. This approach, as has been proposed recently by Medjaher and his co-authors [10], has the advantage that there is no need for elimination of unknown variables from continuity equations in order to obtain ARRs, because a set of equations determining residuals is solved numerically. In general, non-linearities may even prevent the generation of ARRs in symbolic form. In contrast to the method in [10], this paper proposes to couple the process model to the reference model by means of residual sinks introduced in the previous section. Both bond graph models retain integral causality as the preferred causality. Signals from the process model control modulated sources. Their values are compared with corresponding values from the reference model. The process model is subject to faults due to external sources that can be switched on and off. If no faults are introduced into the process model, then the difference between 'measured' signals and their corresponding reference is equal to zero. This difference is input into the residual sinks. If it vanishes, the output of a residual sink is equal to zero. However, if a process variable differs from its reference due to a fault introduced into the process model, the residual sink provides a flow or an effort, respectively in order to adapt the reference model's behaviour to the perturbed process behaviour and to force the difference to zero. This non-zero output of a residual sink is a residual of an ARR and a numerical indicator to a fault. As each junction in the reference model represents a constitutive equation for efforts or flows, respectively, each junction could, in principle, be connected to a residual sink. However, as we already know from section II, residuals may be structurally dependent. Only a subset of them will be needed to build the fault signature matrix. Causal paths between residual sinks will indicate these dependencies. In the next section, this approach is illustrated by means of the often used hydraulic two tanks system.

\section{A Hydraulic Two Tanks Example}

In Fig. 4, the lower part of the overall bond graph represents the process model accounting for faults. The modulated flow sinks, attached to the 0 -junctions, model possible leakage from the tanks, which means that the area of the bottom of the tanks is reduced for some period of time. Representation of the valves by modulated resistors enables to model a blockage of the valves. It is assumed that the pressures in the two tanks are 'measured'. Accordingly, signal arrows are attached to the 0 -junctions. The upper part of the overall bond graph represents the reference model. Both bond graphs are coupled by means of residual sinks (highlighted in red). The right hand side residual effort sink is depicted in grey because it is redundant in the sense that it contributes no fault signature different from those of the other two residuals. Therefore, the residual effort sink is not taken into account in the following. The output of a residual sink, in fact, is a residual of an ARR of a junction. For instance, summing to zero all flows at the left hand-side 0junction of the reference models yields:

$$
\begin{aligned}
f_{1} & =Q_{p}-C_{1} \dot{p}_{1}-Q_{R_{1}} \\
& =Q_{p}-C_{1} \dot{p}_{1}-k_{1} \operatorname{sign}\left(\mathrm{p}_{1}-\mathrm{p}_{2}\right) \sqrt{\left|\mathrm{p}_{1}-\mathrm{p}_{2}\right|}
\end{aligned}
$$

This relation includes only known variables and known parameters and thus, is an ARR.

The two tanks system has been simulated by means of the open source mathematics package Scilab [14]. Formulation of the underlying DAE system in Scilab's mathematical input language is straightforward. The equations can be directly derived from the bond graph of Fig. 4. The Scilab function, $\mathbf{f}$, of the DAE system of the two tanks models coupled by residual sinks is displayed in Fig. 5. The constitutive equations of the energy stores and the equations of the residual sinks have been written in implicit form. The descriptor vector is

$$
\mathbf{x}=\left[p_{1}, p_{2}, \tilde{p}_{1}, \tilde{p}_{2}, f_{1}, f_{2}\right]^{T}
$$

and the DAE system is of the form

$$
\mathbf{A} \dot{\mathbf{x}}+\mathbf{g}(\mathbf{x})=(1,0,1,0,0,0])^{T}\left[Q_{p}\right]
$$

in which $\mathbf{A}$ is a $6 \times 6$, constant coefficient singular matrix and $\mathbf{g}$ a vector function that is nonlinear due to the nonlinear constitutive equations of the orifices. As there are no differential equations for the residuals, $f_{1}, f_{2}$, the last two rows in A vanish. The DAE system is of index $\geq 1$.

For the numerical solution of DAE systems, Scilab provides the widely used DASSL code [18]. In order to facilitate the specification of a consistent set of initial conditions, it is assumed that the tanks are empty at initial time $t=0$ and that the pump delivers a constant volume flow rate, $Q_{p}$, for $10.0 \mathrm{~s} \leq t \leq 40.0 \mathrm{~s}$. That is, the empty tanks are filled 


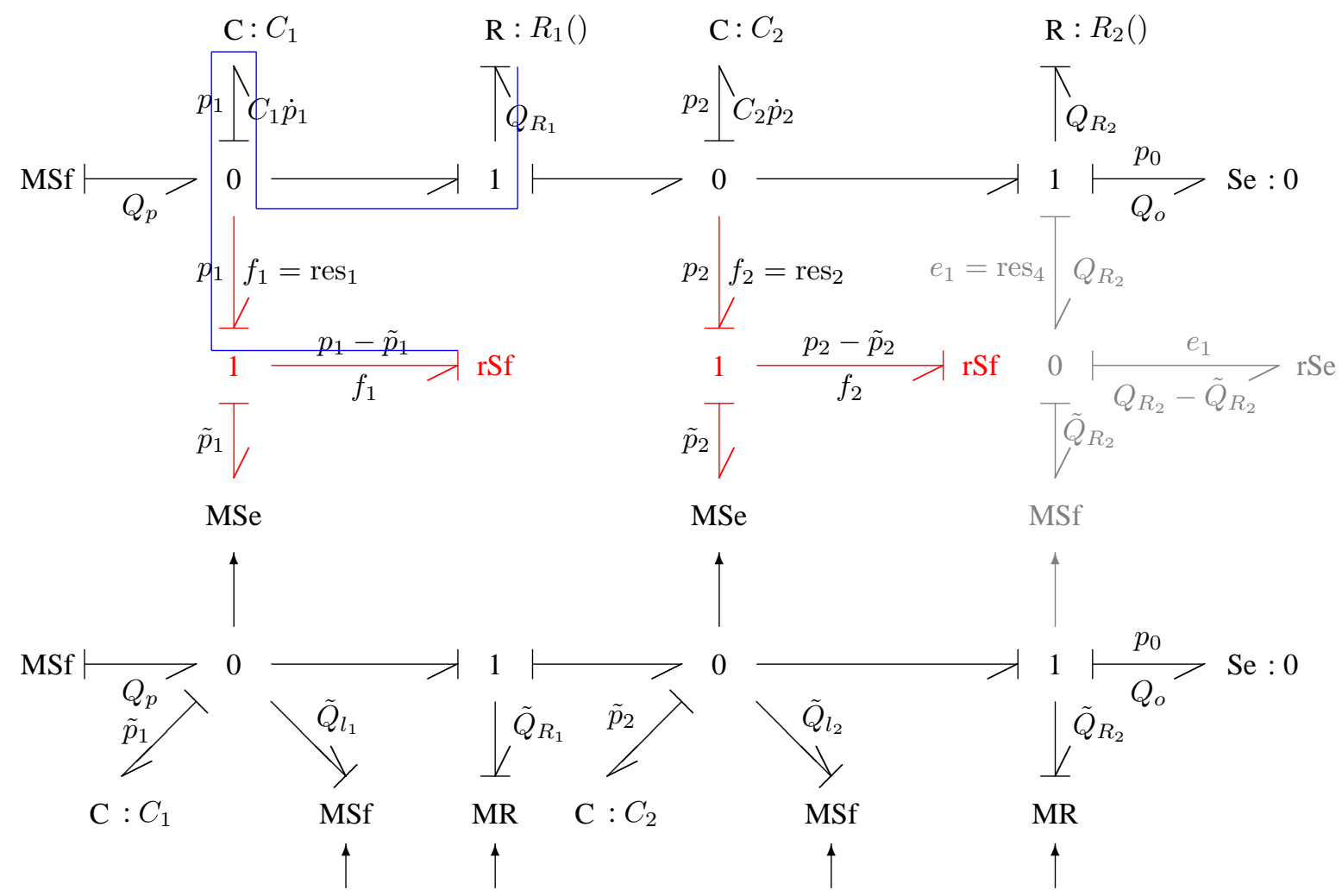

Fig. 4: Coupling of the process model subject to faults (lower part) and the reference model (upper part) by means of residual sinks

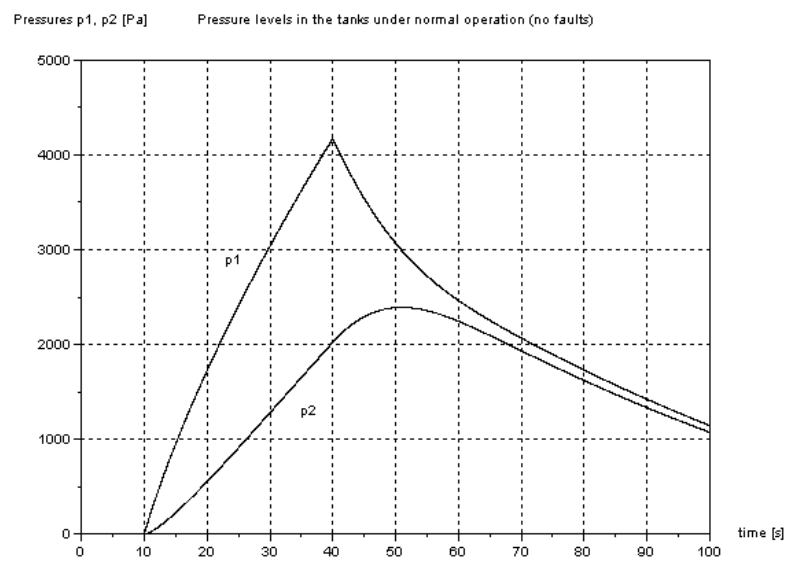

Fig. 6: Pressure levels in the tanks in case of no faults

for 30 s and then they discharge at a rate depending on how much the valves are open. Fig. 6 depicts the undisturbed dynamic behaviour. As a first scenario, a constant leakage flow from tank 1 is assumed to be effective for the time period $50 \mathrm{~s} \leq t \leq 60 \mathrm{~s}$, while the tanks discharge. As a result, the pressures in the tanks decrease at a higher rate during this time period. Fig. 7 shows the time evolution of the tank pressures in the case of a leakage from tank 1. A leakage from tank 1 corresponds to a decrease of the area of its bottom. According to the fault signature matrix, residual res ${ }_{1}$ is affected, while residual res $_{2}$ is not. Figures 8 and 9 depict the residuals $f_{1}$ and $f_{2}$. Another fault that may occur is a partial blockage of the valve between the two tanks. If it happens after the pump has switched off, then, during the time period this fault is effective, the pressure in the first

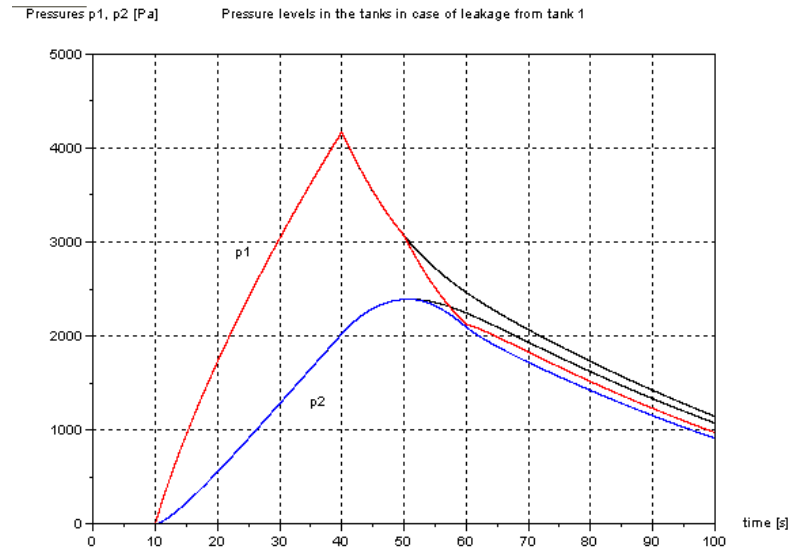

Fig. 7: Time history of the tank pressures in case of a leakage from tank 1

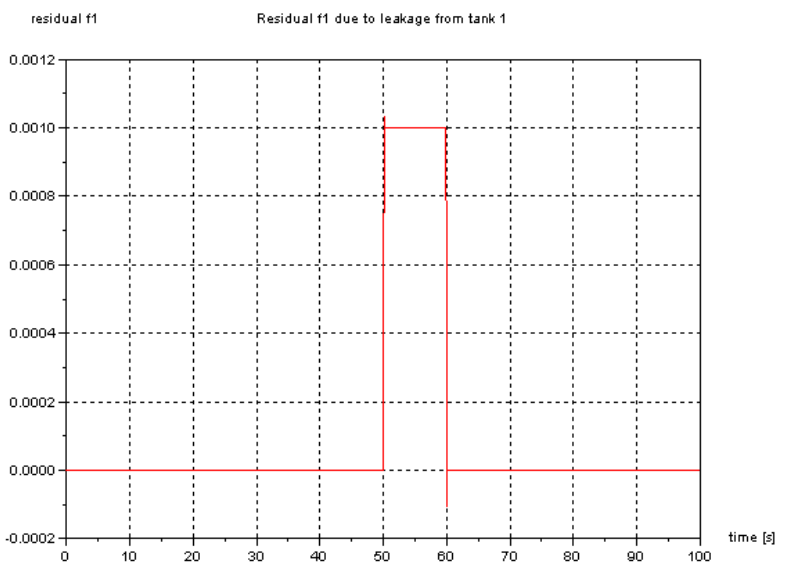

Fig. 8: Residual $f_{1}$ in case of a leakage from tank 1 




Fig. 5: Scilab function with the DAE system of the two tanks models coupled by residual sinks

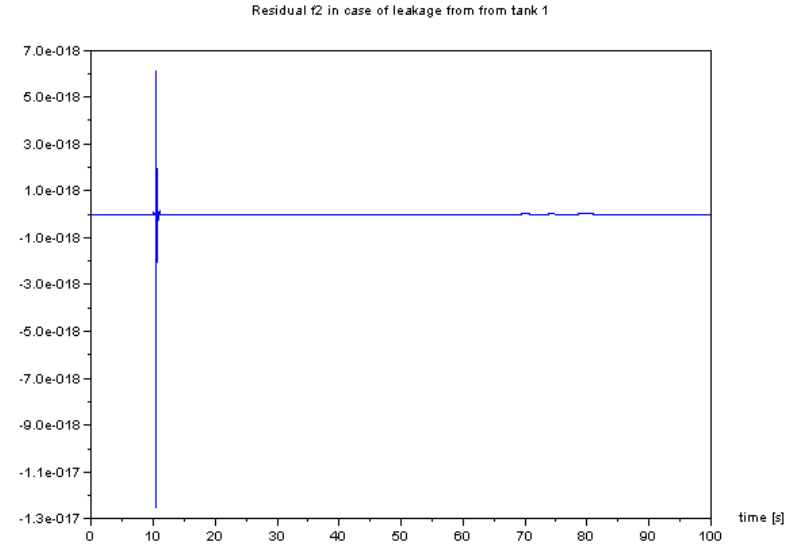

Fig. 9: Residual $f_{2}$ in case of a leakage from tank 1

tank decreases at a lower rate, while the pressure in the second tank decreases at a higher rate. This can be seen from Fig. 10. According to the fault signature matrix both residuals are sensitive to a fault in valve 1 connecting both tanks.

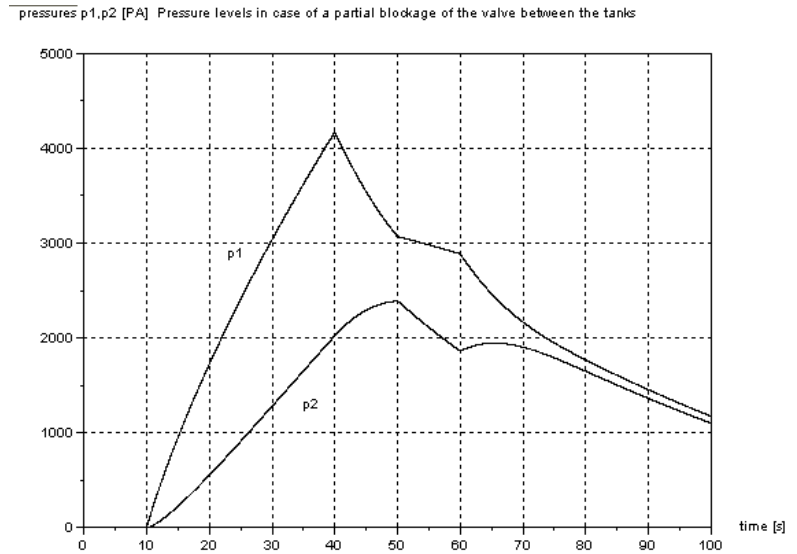

Fig. 10: Pressure levels in the two tanks in case of a partial blockage of the valve between the tanks

This is verified by Fig. 11. Finally, Fig. 12 shows the numerical values of the residual $f_{2}$ for the cases of leakage from tank 2 and a partial blockage of valve 2 . In accordance with the fault signature matrix, residual $f_{2}$ is sensitive to these 




Fig. 11: Residuals $f_{1}$ and $f_{2}$ due a partial blockage of the valve between the tanks



Fig. 12: Residual $f_{2}$ due to leakage from tank 2 (positive values) and a partial blockage of valve 2 (negative values)

faults.

\section{Direct Determination of Fault Signatures FROM THE BOND GRAPH}

Computation of the two models coupled by means of residual sinks provides numerical values for the residuals of the ARRs as has been illustrated in the previous section. Moreover, analysis of signal forward paths in the reference model part can directly reveal the entries in the fault signature matrix [11]. In fact, there is, for instance, a causal path between the resistor of valve 1 and the residual sink providing res $_{1}$, which can be indicated by the sequence of power variables involved:

$$
R_{1} \rightarrow Q_{R_{1}} \rightarrow C_{1} \dot{p}_{1} \rightarrow C_{1} \rightarrow p_{1} \rightarrow\left(p_{1}-\tilde{p}_{1}\right) \rightarrow \mathrm{rSf} \rightarrow f_{1}
$$

(Fig. 4). In other words, the volume flow rate $Q_{R_{1}}$ through valve 1 depending on the valve's cross sectional area contributes to the sum of flows at the left-side 0-junction. Consequently, valve 1 contributes a non-zero entry to the signature of the ARR of residual res 1 and, for the reason of symmetry, also a '1' to the signature of the ARR of residual $\mathrm{res}_{2}$. Furthermore, there are causal paths between a $\mathrm{C}$ energy store and a residual sink, e. g.:

$$
C_{1} \rightarrow p_{1} \rightarrow\left(p_{1}-\tilde{p}_{1}\right) \rightarrow \mathrm{rSf} \rightarrow f_{1}
$$

This is an indication that tank i contributes a non-zero entry to the signature of the ARR of residual res $\mathrm{r}_{\mathrm{i}},(i=1,2)$. From the fault signature matrix in Table I we already know that $p_{2}$ also contributes to the signature of the ARR of residual res ${ }_{1}$. In fact, there is a causal path between the $C$ energy store of the second tank and the resistor of valve 1 . The output of this resistor, $Q_{R_{1}}$, is part of the sum of all flows at the leftside 0 -junction. Consequently, the pressure $p_{2}$ contributes to the ARR of $\mathrm{res}_{1}$. By this way, all non-zero entries in the fault signature matrix can be identified directly from the reference model part of the overall model.

Finally, Fig. 4 shows that the output of the right hand side residual effort sink, $e_{1}=$ res $_{4}$, is converted into the volume flow $Q_{R_{2}}$, which in turn contributes to the sum of flows at the 0 -junction representing the pressure $p_{2}$ in the tank $T_{2}$.

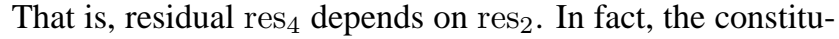
tive equation of the outlet valve of tank $T_{2}$ reads:

$$
Q_{R_{2}}=k_{2} \operatorname{sign}\left(\mathrm{p}_{2}-\mathrm{res}_{4}\right) \sqrt{\left|\mathrm{p}_{2}-\mathrm{res}_{4}\right|}
$$

On the other hand, the sum of flows at the 0 -junction representing $p_{2}$ yields:

$$
\begin{aligned}
Q_{R_{2}} & =Q_{R_{1}}-C_{1} \dot{p}_{2}-\operatorname{res}_{2} \\
& =k_{1} \operatorname{sign}\left(\mathrm{p}_{1}-\mathrm{p}_{2}\right) \sqrt{\left|\mathrm{p}_{1}-\mathrm{p}_{2}\right|}-\mathrm{C}_{1} \dot{\mathrm{p}}_{2}-\operatorname{res}_{2}(8)
\end{aligned}
$$

Hence, res $_{4}$ provides no fault signature different from the



\section{CONCLUSIONS}

Model based fault detection and isolation builds on the comparison of data from a process engineering system with data from a reference model. However, the introduction of faults into a real world process for test purposes can be impossible or unwanted for various reasons. This suggests to replace the real process by a model that can include all kinds of faults that are of interest at no risk. This paper proposes to retain preferred integral causality in both models and to couple them by means of residual sinks that have been in use for various other purposes also. These artificial elements play a clear and intuitive role. They provide an effort or a flow that forces the difference between a monitored variable and its reference to zero and that adapts the reference to the signal perturbed by a fault. At the same time, the output of the residual sink is a residual of an ARR. Simulation of the two process models coupled by residual sinks enables a numerical evaluation of the residuals of ARRs. For computation of the underlying DAE system determining the residuals, the well known DASSL code can be used. Simulation experiments have shown that parameters controlling the numerical integration should be chosen with care. For instance, in case the Jacobian matrix has not been provided in symbolic form, values for absolute and relative error tolerances, that have been chosen too small, can lead to a failure of the computation. Such problems with the numerical solution of DAE systems are well known. On the other hand, in the simulation experiments carried out, numerical errors clearly have shown to be orders of magnitude smaller than the values of the residuals so that the latter could be clearly distinguished from numerical errors. As the model including faults represents the real world process, values of 'monitored' process variables can be superimposed by noise before they are 
used as an input into a residual sink. While algebraic loops and non-linearities in a model may prevent the generation of ARRs in symbolic form, numerical solution of DAE system determining the residuals of ARRs is mostly feasible. Moreover, inspection of the reference model part with regard to causal paths ending at residual sinks enables to set up the fault signature matrix directly from the bond graph, i. e., equations do not need to be formulated and manipulated.

The two process models coupled by residual sinks can serve for the analysis of various fault scenarios. One advantage of using a model for the faulty real process is that signals from the simulated process may be obtained much quicker than in real time.

\section{REFERENCES}

[1] D.C. Karnopp, D.L. Margolis, and R.C. Rosenberg. System Dynamics - Modeling and Simulation of Mechatronic Systems. John Wiley \& Sons Inc., Third edition, 2000. ISBN: 0-471-33301-8.

[2] G. Dauphin-Tanguy. Les bond graphs. Hermes Science Europe Ltd., Paris, France, 2000. ISBN: 2-7462-0158-5.

[3] A. Mukherjee and R. Karmakar. Modelling and Simulation of Engineering Systems through Bondgraphs. Narosa Publishing House, New Delhi, India, 2000. ISBN: 81-7319-279-0.

[4] W. Borutzky. Bond graphs - A Methodology for Modelling Multidisciplinary Dynamic Systems, volume FS-14 of Frontiers in Simulation. SCS Publishing House, Erlangen, San Diego, 2004. ISBN: 3-936150-33-8.

[5] J. Thoma and B. Ould Bouamama. Modeling and Simulation in Thermal and Chemical Engineering (A Bond Graph Approach). SpringerVerlag, 2000.

[6] C. Heny, D. Simanca, and M. Delgado. Pseudo-bond graph model and simulation of a continuous stirred tank reactor. Journal of The Franklin Institute, 337:21-42, 200.

[7] P. J. Feenstra, P. J. Mosterman, G. Biswas, and P. C. Breedveld. Bond Graph Modeling Procedures for Fault Detection and Isolation of Complex Flow Processes. In J. J. Granda and G. Dauphin-Tanguy, editors, Proc. of the International Conference on Bond Graph Modeling, ICBGM'01, pages 77-82. SCS Publishing, January 7-11 2001. Simulation Series, Vol. 33, Number 1, ISBN: 1-56555-221-0.

[8] B. Ould Bouamama, A. K. Samantaray, M. Staroswiecki, and G. Dauphin-Tanguy. Derivation of Constraint Relations from Bond Graph Models for Fault Detection and Isolation. In J. J. Granda and F. Cellier, editors, Proc. of the International Conference on Bond Graph Modeling, ICBGM'03, pages 104-109. SCS Publishing, January 1923 2003. Simulation Series, Vol. 35, Number 2, ISBN: 1-56555-2571 .

[9] K. Sia and A. Naamane. Bond Graph: a suitable tool for component faults diagnostic. In J. J. Granda and F. Cellier, editors, Proc. of the International Conference on Bond Graph Modeling, ICBGM'03, pages 89-103. SCS Publishing, January 19-23 2003. Simulation Series, Vol. 35, Number 2, ISBN: 1-56555-257-1.

[10] K. Medjaher, A. K. Samantaray, and B. Ould Bouamama. Diagnostic Bond Graphs for Direct Residual Evaluation. In J. J. Granda and F. Cellier, editors, Proc. of the International Conference on Bond Graph Modeling, ICBGM'05, pages 307-312. SCS Publishing, January 2327 2005. Simulation Series, Vol. 37, Number 1, ISBN: 1-56555-2873.

[11] S. K. Ghoshal, A. K. Samantaray, and A. Mukherjee. Improvements to Single Fault Isolation Using Estimated Parameters. In J. J. Granda and F. Cellier, editors, Proc. of the International Conference on Bond Graph Modeling, ICBGM'05, pages 301-306. SCS Publishing, January 23-27 2005. Simulation Series, Vol. 37, Number 1, ISBN: 156555-287-3.

[12] W. Borutzky and F. E. Cellier. Tearing algebraic loops in bond graphs. TRANSACTIONS of the SCS, 13(2):102-115, June 1996.

[13] W. Borutzky. Supporting the generation of a state space model by adding tearing information to the bond graph. Simulation Practice and Theory, 7(5-6):419-438, 1999.

[14] Scilab Consortium. Scilab. http://www.scilab.org/.

[15] A. M. Bos. Modelling Multibody Systems in Terms of Multibond Graphs with Application to a Motorcycle. PhD thesis, Univ. of Twente, Enschede, The Netherlands, 1986.

[16] D. Karnopp. Lagrange's equations for complex bond graph systems. ASME Journal of Dynamic Systems, Measurement, and Control, 99(4):300-306, December 1977.

[17] P. Gawthrop and L. Smith. Causal augmentation of bond graphs with algebraic loops. Journal of the Franklin Institute, 329:291-303, 1992.

[18] K. E. Brenan, S. L. Campbell, and L. R. Petzold. Numerical Solution of Initial-Value Problems in Differential-Algebraic Equations. NorthHolland, 1989.

\section{APPENDIX}

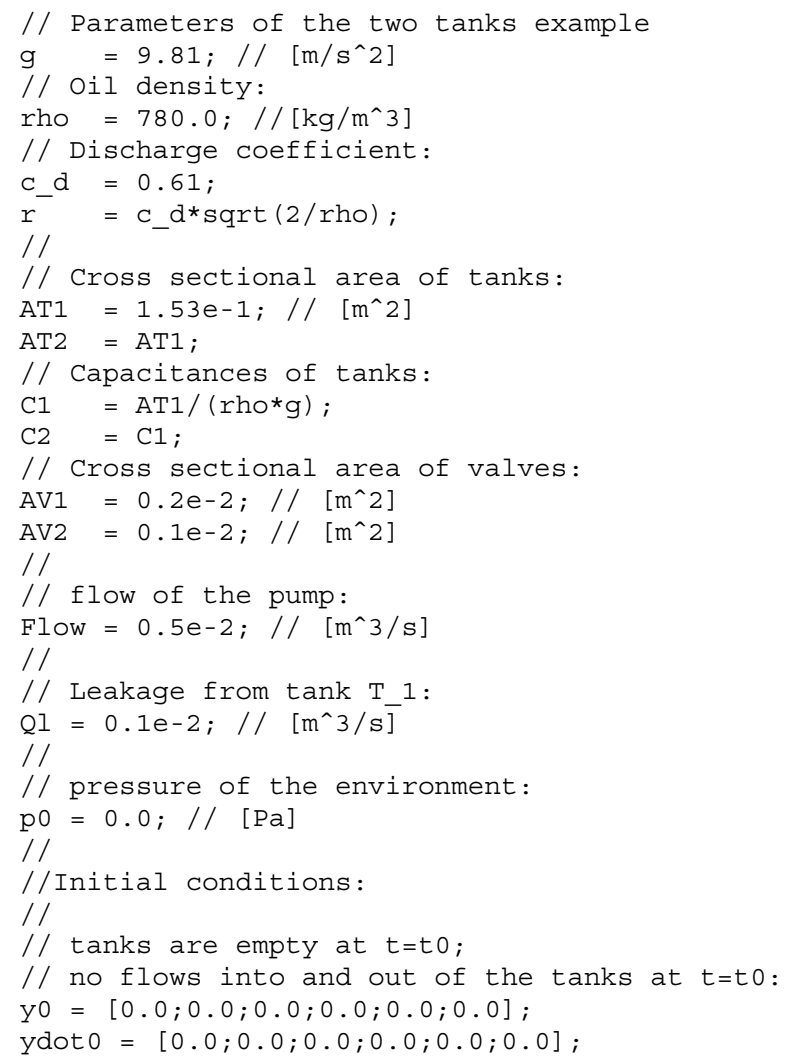

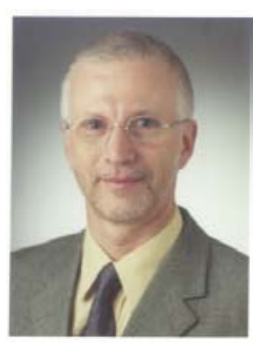

(2001, 2002)

His main scientific interests have been modelling and simulation methodologies for multidisciplinary systems, especially Bond Graph based as well as object oriented modelling; modelling, simulation, control and fault diagnosis of mechatronic systems, modelling languages, software design for modelling and simulation of continuous, as well as hybrid systems, web-based simulation, scientific computing, numerical algorithms and software design for (parallel) continuous system simulation.

Dr. Borutzky has published extensively in major international conferences on Modelling and Simulation and in refereed scientific journals. He is author of the monograph "Bond Graphs - A Methodology for Modelling Multidisciplinary Dynamic Systems" (SCS Publishing House, Erlangen, San Diego, 2004).

Since 1990 he has served in many international scientific conferences on Modelling and Simulation in various positions, in 2005 as Assistant General Chair of the European Conference on Modelling and Simulation (EMCS) in Riga, Latvia and as General Chair of the ECMS 2006 in Sankt Augustin, Germany.

Dr. Borutzky is a member of ASIM, a senior member of SCS and currently serves on the BoD of SCS. He is also active as a member of the Editorial Board of some simulation related journals.

E-mail address: borutzky@uni-koeln.de,web-page: http://www2.inf.fhbrs.de/ wborut $2 \mathrm{~m} /$ 